\title{
Proposal of A Dynamic Channel Allocation Method for MIMO-OFDMA System
}

\author{
Quoc Khuong Nguyen ${ }^{1}$, Van Duc Nguyen ${ }^{1}$, Quoc Trung Nguyen ${ }^{1}$, Minh Hung Dao ${ }^{2}$ \\ ${ }^{1}$ Faculty of Electronic and Telecommunication Hanoi University Of Technology, \\ No 1 - Dai Co Viet - Hanoi \\ Email:nqkhuong@mail.hut.edu.vn \\ ${ }^{2}$ Faculty of Technique and Technology Quynhon University, 170 An Duong Vuong, \\ Quy Nhon City, Binh Dinh Province
}

\begin{abstract}
This paper proposed a dynamic channel allocation method for MIMO-OFDMA system, which can be applied for both uplink and downlink. The SNR on each subcarrier linking from a BS to all active MSs is estimated by ZF and MMSE approach. Only subcarriers having maximum SNR are selected for the corresponding communication link. The proposed algorithm is investigated for different system parameters and the channel conditions. Numerical results show that the system performance can be significantly improved by applying the proposed technique.
\end{abstract}

Keyword: MIMO-OFDMA, DCA (Dynamic Channel Allocation), ZF (Zero Forcing), MMSE (Minimum Mean Square Error)

\section{INTRODUCTION}

Recently, wireless communication using the Multiple Input Multiple Output - Orthogonal Frequency Division Multiple Access (MIMOOFDMA) technique has attracted much research interest. This is due to the fact that the MIMO technology increases the system capacity. OFDM techniques is a multicarrier-based technique for alleviating inter symbol interference (ISI) by transforming the frequency selective channel into a set of flat fading channels. Thus, the combination of MIMO-OFDM offers a system with the high data rate.

MIMO-OFDM has been used in many multiuser environments like wireless LAN (IEEE802.11), Wimax (IEEE802.16). In these systems, the users must share the system bandwidth to the other users. There are some traditional methods that have been applied in practice, such as TDMA, FDMA, CDMA, CSMA/CA. In a conventional OFDM system, each user uses all subcarriers for its transmission. Therefore, this method is not dynamic to assign bandwidth for different users. The OFDMA channel allocation method allows multiple users to share the subcarriers for simultaneous transmission. Thus, OFDMA technique adds another dimension for channel allocation, and increases the throughput of the network.

Comparing to TDMA, an MS using OFDMA method can transmit or receive data simultaneously to or from BS, respectively. Thus, the guard time in TDMA mode user separation can be removed. In other side, in Rayleigh fading channel with the time and frequency selectivity, a user will experience a deep fade in several particular subcarriers. In the case that the channel information is known by transmitter, only "good subcarriers" are assigned to a user in a particular time duration.

Efficient channel allocation for increasing system performance has been investigated in literature[1-6]. In [1] Ying proposed an adaptive resource-allocation algorithm for dynamic subcarrier selection based on optimization power, bit rate and modulation level. This algorithm, however, requires high complexity computation. In [2], [3] the rate-adaptive problem was tackled, wherein the objective was to maximize the total data rate over al. users subject to power and BER constraints. In [4], Nguyen et al. proposed a novel decentralized dynamic channel assignment (DCA) 
algorithm for an OFDM network operating in time division duplexing (TDD) mode with full frequency reuse. The key of channel allocation is to reduce the co-channel interference (CCI) and to increase the overall throughput of the network. Some other authors use water filling method to achieve maximum system capacity for all users [5][6]. Wong et al in [7] use Lagrange method to optimize the transmitting power under the condition of bit rate constrain.

One of difficulties of MIMO-OFDMA system is the synchronization for MSs and BS in uplink communication. The frequency and phase offset always exist in OFDM system, which causes the intercarrier interference. This major issue is more serious in multiuser environments.

In this paper, we propose a dynamic channel allocation method for MIMO-OFDMA networks. The proposed method can be used for both uplink and downlink. In the uplink communication, channel is estimated by BS. In downlink, the channel information can be obtained by the low rate feedback channel. Based on the estimated SNR, only the subcarriers with high SNR are assigned to the corresponding users.

The MIMO system capacity depends on channel correlation, the number of transmitting and receiving antennas. The spatial positions of antenna assembled in MS and BS affect channel correlation. In practice, the channel correlation must be taken into account. However, to simplify the complexity of the proposed algorithm and without loss of generality, we assume that the MIMO channel are uncorrelated.

The paper is organized as follows: Section II introduces the MIMO-OFDMA system briefly. Section III describes the proposed algorithm. Simulation results are presented in Section VI. Section $\mathrm{V}$ remarks conclusions.

\section{SYSTEM MODEL}

We consider a MIMO-OFDMA system including a $B S$ equipped with $\mathrm{Q}$ antennas and $M$ users (MS) with $L$ antennas.
Let us denote $\mathbf{H}_{k}^{i}$ the transmit channel matrix size $(Q \times L)$ at subcarrier $k^{\text {th }}$ between $L$ transmit antennas of $M S_{i}$ with Q receive antennas of $B S$.

Assuming that the channel is known at both transmitter and receiver by the feedback channel.

Before transmitting data, the data of a MS is converted from serial to parallel, and is carried on a set of subcarrier set $\left\{C^{i}\right\}$. The set $\left\{C^{i}\right\}$ was selected by $\mathrm{BS}$, then this information is transferred to the MS by the feedback channel (see Fig. 1).

All subcarriers, which do not belong to set $\left\{C^{i}\right\}$, are set to zero. The data on the selected subcarrier is led to the OFDM modulator and transferred to $L$ antennas for transmission (Fig. 1) .

The symbol $\vec{S}_{k}^{i}$ is a vector of size $(L \times 1)$, which is the transmitted signal of $M S_{i}$ at the $\mathrm{k}^{\text {th }}$ subcarrier. $\vec{Y}_{k}^{i}$ is a vector of size $(Q \times 1)$ which is the received signal at $\mathrm{BS}$ of $M S_{i}$ at the $\mathrm{k}^{\text {th }}$ subcarrier. The received signal can be written as follows:

$$
\vec{Y}_{k}^{i}=\sqrt{\frac{E_{s}}{L}} \mathbf{H}_{k}^{i} \vec{S}_{k}^{i}+\vec{N}_{k}^{i}
$$

where $\vec{N}_{k}^{i}$ is the Gaussian noise vector with size of $(Q \times 1) . \quad E_{s}$ is the total signal power emitting from $L$ transmit antennas

By using the zero forcing method (ZF) demodulation, the demodulated symbol is given as follows

$$
\vec{S}_{k}^{i, Z F}=\left(\left(\left(\mathbf{H}_{k}^{i}\right)^{H} \mathbf{H}_{k}^{i}\right)^{-1}\left(\mathbf{H}_{k}^{i}\right)^{H}\right) \vec{Y}_{k}^{i}
$$

In [9], the signal to noise ratio at receiver for the case of MIMO channel using ZF method meet the following condition

$$
\operatorname{SNR}\left(\mathbf{H}_{k}^{i}\right)^{Z F} \geq \lambda_{\min }\left(\mathbf{H}_{k}^{i}\right) \frac{E_{s}}{L N_{0}}
$$

where $\lambda_{\min }$ is the minimum singular value of the $\mathbf{H}_{k}^{i}$ channel matrix.

The demodulated symbol obtained by using MMSE filter is written as follow [9] 


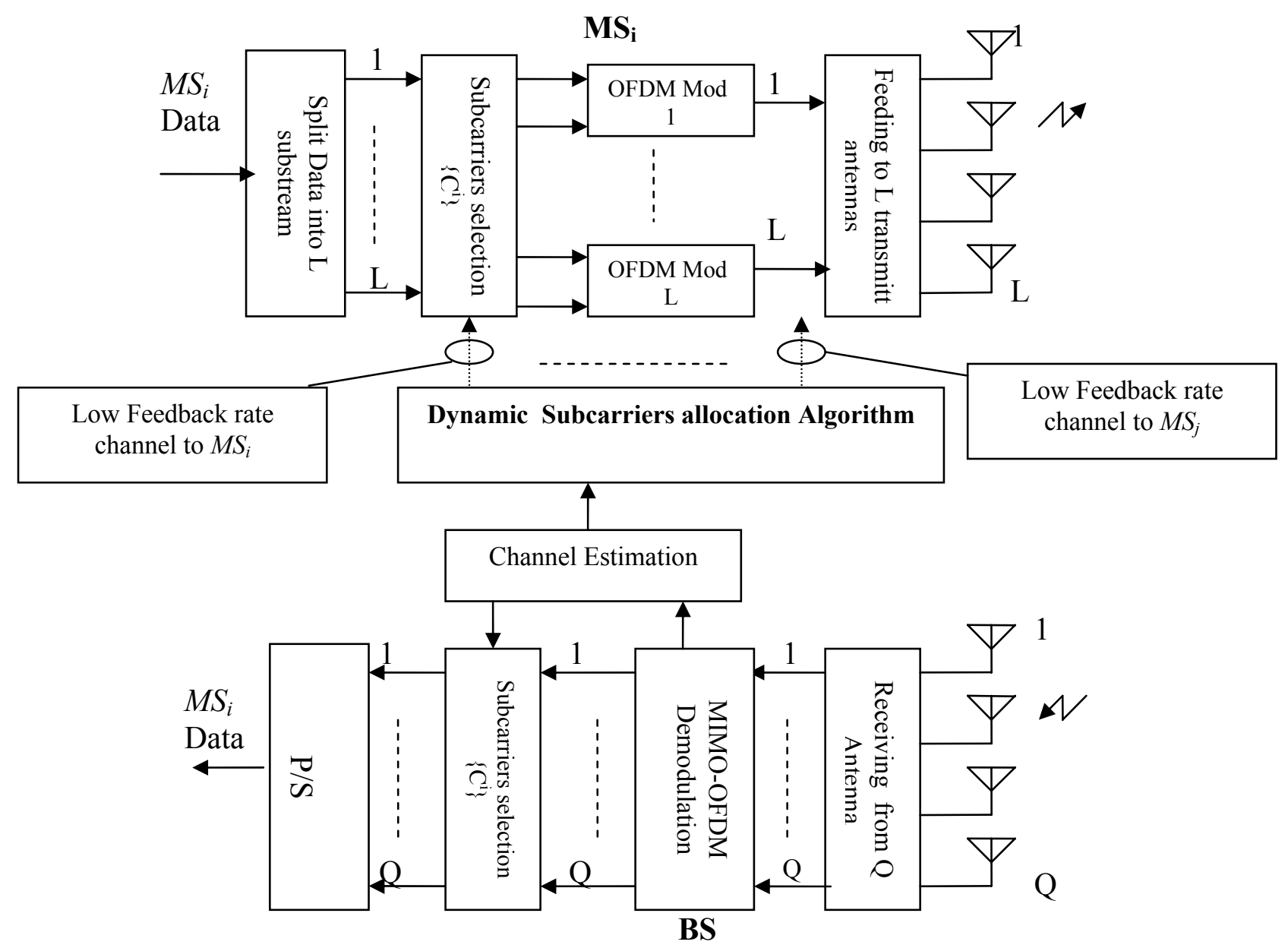

Figure 1: MIMO-OFDMA System

$$
\vec{S}_{k}^{i, M M S E}=\left(\left(\mathbf{H}_{k}^{i}\right)^{H} \mathbf{H}_{k}^{i}+\frac{N_{0}}{E_{S}} \mathbf{I}_{L}\right)^{-1}\left(\mathbf{H}_{k}^{i}\right)^{H} \vec{Y}_{k}^{i}
$$

where $\mathbf{I}_{L}$ is unit matrix with a size of $(L \times L)$, and $\mathbf{H}^{H}$ is the Hermitian channel matrix.

The SNR for MMSE case can be calculated by

$$
\operatorname{SNR}\left(\mathbf{H}_{k}^{i}\right)^{M M S E}=\frac{E_{s}}{L N_{0}\left(\mathbf{H}_{k}^{i} * . \mathbf{H}_{k}^{i}+N_{0} / E_{s} \mathbf{I}_{L}\right)^{-1}}-1
$$

We assume that MS distribution is random and uniform in a cell. Transmitting power from all MSs to $\mathrm{BS}$ is equal. The channel between MS and BS is timeand frequency-selective and follows the Rayleigh distribution.

MAC frame structure for MIMO-OFDMA system is depicted in Fig 2. The preamble field SYN is used to synchronize between MS and BS. TG is delay time, which is reserved to change the mode of uplink and downlink transmission. The registration process for a MS is carried out in the "random access" time slot of each MAC frame. In this time slot, the registration protocol for a user accessing the network follows the CSMA/CA 802.11 mechanism.

\begin{tabular}{|l|l|l|l|l|}
\hline SYN & DOWNLINK & TG & UPLINK & $\begin{array}{l}\text { Random } \\
\text { Access }\end{array}$ \\
\hline
\end{tabular}

\section{Figure 2: MAC Frame structure}

We assume that each $M S$ can have maximum $P$ subcarriers. Of course, $\mathrm{P} \leq N$, where $\mathrm{N}$ is the total number of subcarriers. The choice of $P$ value must be taken into account, because this parameter will influence on the system performance. In the case of a small $P$ value, more MSs have chance to access to the network at the same time, but less opportunities to get "good" sub-carriers. We can prove that the system performance will be increased, if we increase $P$. This 
is because, in the case of a larger $P$ value, there are more chances to assign "good" sub-carriers to MSs entering the network. Therefore, system throughput can be increased. To achieve the maximum system performance by using the proposed algorithm, we set $P=N$ for all simulations.

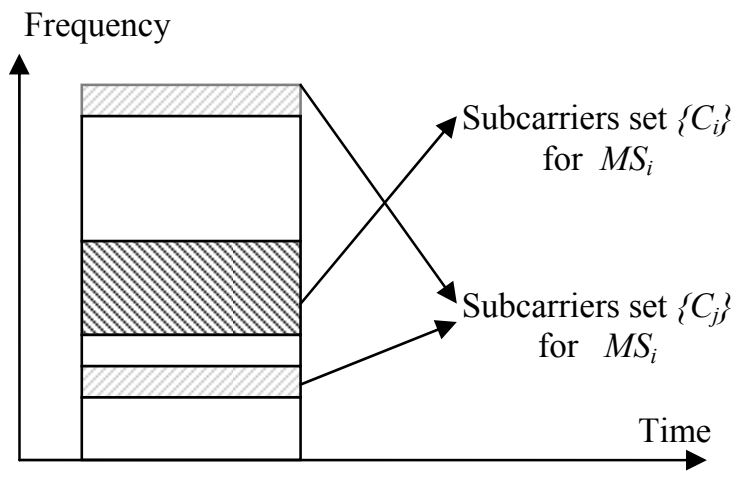

Figure 3: Subcarrier allocate for MS

Note that $C^{i}=\left\{c_{1}^{i}, c_{2}^{i}, \ldots ., c_{N}^{i}\right\}$ is the set of all subcarriers, which are assigned for $M S_{i}$ (Fig 3). The value of $c_{k}^{i}$ is assigned by the following condition $c_{k}^{i}= \begin{cases}1 & \text { If the } \mathrm{k}^{\text {th }} \text { subcarrier is assigned for } M S_{i} \\ 0 & \text { Otherwise }\end{cases}$ where $k \in\{1, \ldots N\}$

For M users, it is clear that $\sum_{i=1}^{M} \sum_{k=1}^{N} c_{k}^{i} \leq N$.

III. PROPOSAL OF A DYNAMIC CHANNEL ALLOCATION FOR MIMO-OFDMA SYSTEM

DCA algorithm is proposed on basis of the SNR calculated on each subcarrier. We assume that BS knows full CSI information between BS and MS. The channel allocation is performed periodically per $\mathrm{Z}$ MAC frames.

The proposed algorithm (Fig 4) is described in the following steps.

- Step 1: For the first frame $F=1$, set all elements of $\left\{C^{i}\right\}=0$.

- Step 2: BS measures all channels from all MSs entering to the network. For each subcarrier $k^{\text {th }}$,
BS will calculate the $\operatorname{SNR}\left(\mathbf{H}_{k}^{i}\right)$ in Eq. (3) or (5) for all MSs.

- Step 3: The most suitable MS, which has the maximum of $\arg \left(\operatorname{SNR}\left(H_{k}^{i}\right)\right)$, for $i=1, \ldots, M$, is selected, i.e.

$$
\begin{gathered}
i_{\text {opt }}=\arg _{i}\left(\max \left(\operatorname{SNR}\left(H_{k}^{i}\right)\right)\right) \\
i=1, \ldots, M
\end{gathered}
$$

and the corresponding element $c_{k}^{i_{o p t}}$ is set to 1 .

- Step 4: The summation of the set $\left\{C^{i}\right\}$, i.e $\operatorname{sum}\left\{C^{i}\right\}$, is the total subcarriers assgined for $M S_{i}$

If $\operatorname{sum}\left(C^{i}\right) \geq P$, then $M S_{i}$ is refused to have a new subcarrier.

- Step 5: From $2^{\text {th }}$ to $Z^{\text {th }}$ MAC frame, the set of selected subcarriers is unchanged. MSs use the set $\left\{C^{i}\right\}$ to communicate with BS.

From the $(\mathrm{Z}+1)^{\text {th }}$ MAC frame, steps 1-5 are repeated.

\section{SIMULATION RESULTS AND DISCUSSIONS}

The simulation system includes $1 B S$ and M MSs with 2 transmit and 2 receiver antennas. For simulations, $\mathrm{M}$ can be assigned by 1,2,5, or 10 . MIMO Channel Rayleigh fading is considered uncorrelated and is simulated by Monte-Carlo method with parameters adopted from the HiperLAN/2 channel [8]. Simulation channel is indoor channel [8].

The time response of channel is $400 \mathrm{~ns}$, which corresponds to channel length $\mathrm{L}=16$ sampling intervals. The guard interval (GI) length of an OFDM symbol is 20 sampling intervals. The OFDM system has 64 subcarriers, and uses the 16-QAM. Assuming that the channel is perfectly known at receiver, both ZF and MMSE method are implemented for comparison.

The simulation results in Fig.5 show that the ZF method slightly outperforms the MMSE method at high SNR. In low range of SNR, ZF and MMSE perform nearly the same results. In the following, the 
$\mathrm{ZF}$ is used to verify the proposed algorithm performance.

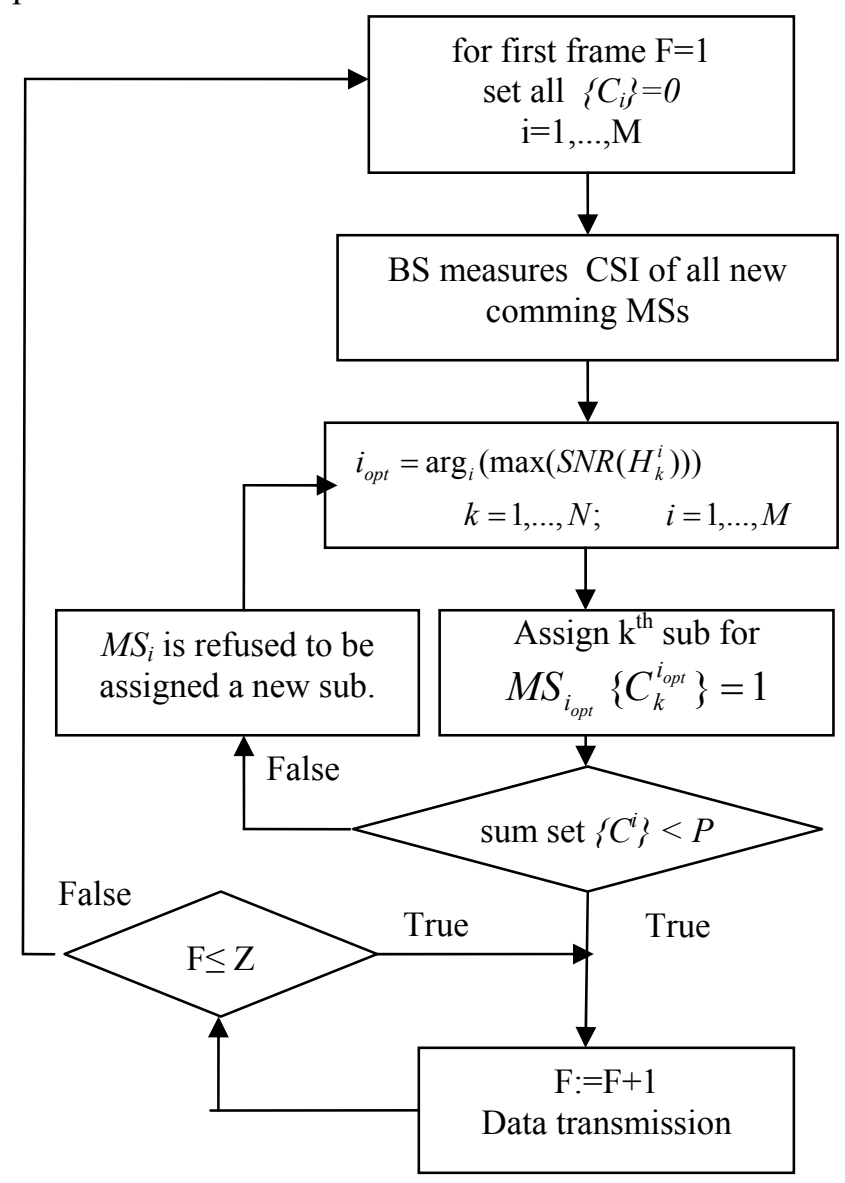

Figure 4: DCA Algorithm scheme

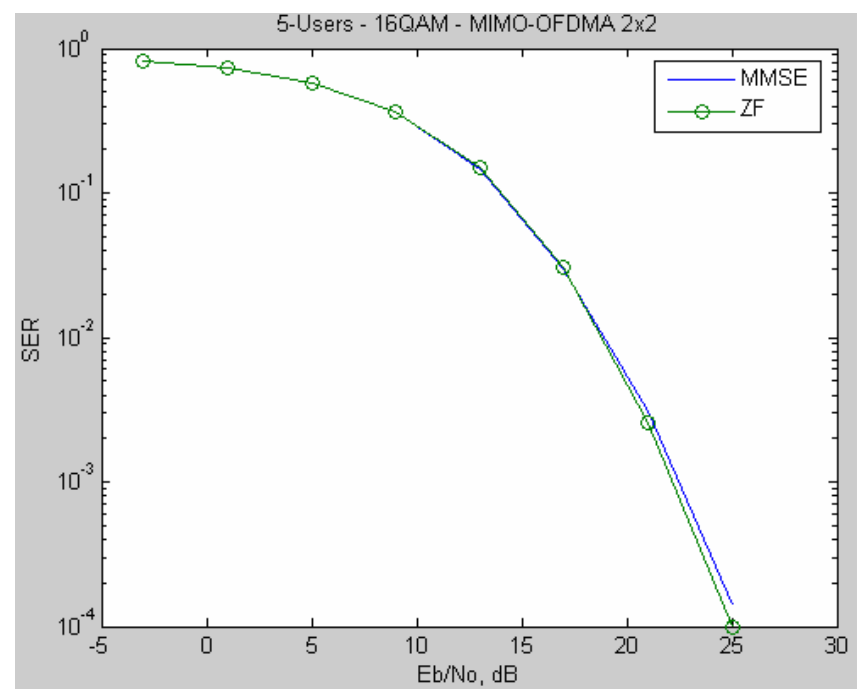

Figure 5: Comparison ZF and MMSE

Fig.6 shows that the system has lowest performance for the case of 1 user. This case is equivalent to the case that the proposed algorithm is not applied. If 2 users request for data transmission, then we will have the diversity regarding the SNR on each sub-carriers for that users. The proposed algorithm takes its advantages in allocating sub-carriers. The system performance is therefore improved. This effect is maintained when we increases the number of users. However, the SNR diversity resource reaches an upper bound, if we continue to increase the number of users. In other words, the difference of SNR on subcarriers in the case of high number of users is not significant as that of the case of only few numbers of users. This reason explains for the tendency of the simulation results while increasing the number of users.

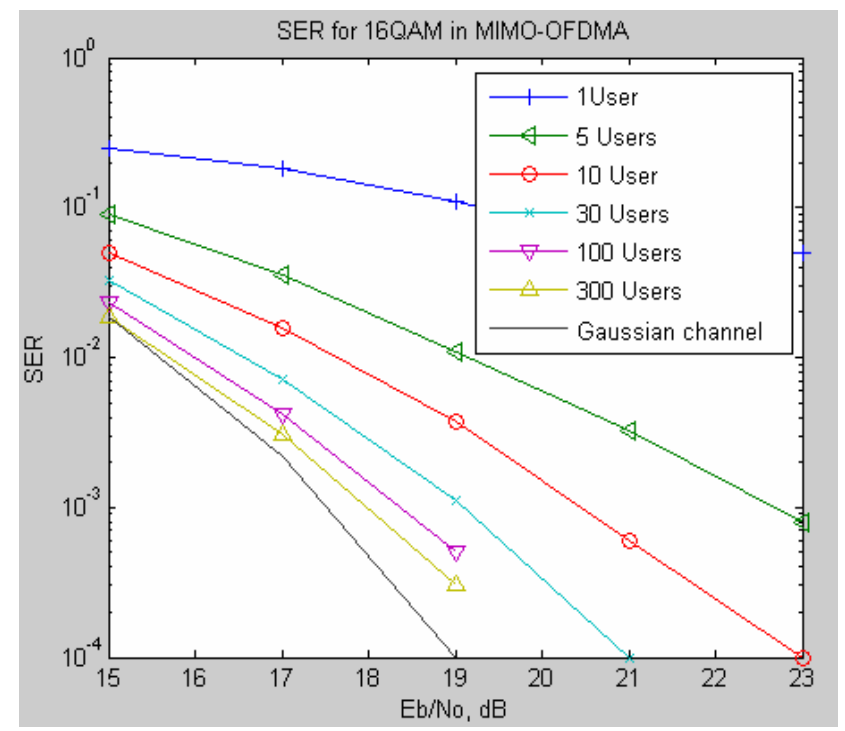

Figure 6: SER in the case of different numbers of users

Fig.7 shows the influence of the Doppler frequency on the network performance. Clearly, the Doppler frequency impacts on the system performance. Similarly, number of symbols per MAC frame affects also system performance (see Fig. 8). This is, because duration of MAC frame should be designed to be smaller than channel correlation time to maintain the system performance.

\section{CONCLUSIONS}

A dynamic channel allocation algorithm for MIMOOFDMA system is proposed in this paper. This 
method has been investigated for different system and channel parameters, as well as for both ZF and MMSE equalization techniques. Numerical results show that a good system performance can be obtained, if the selection of subcarriers can be optimized to have high SNR on each selected subcarriers.

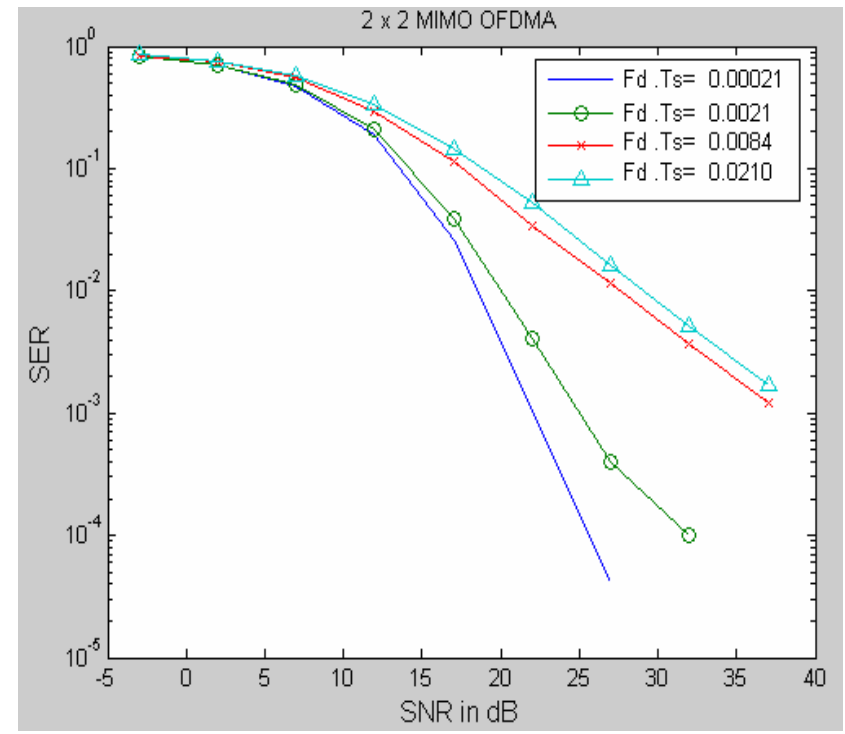

Figure 7: 5 users and 50 Symbol per MAC frame are taken into account; The system is considered for different Doppler frequency

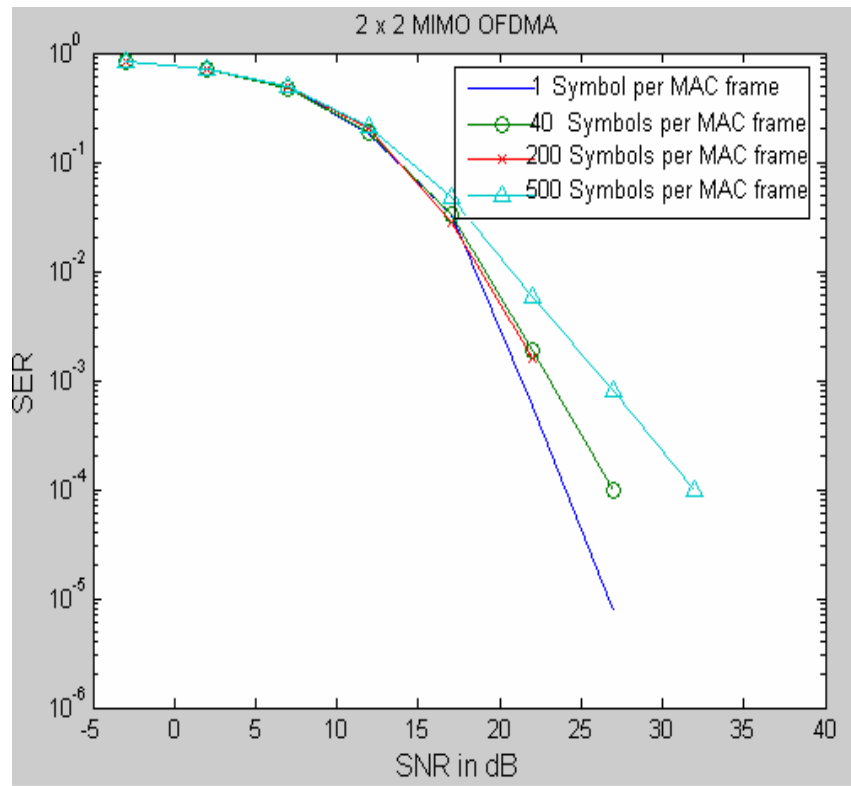

Fig 8: In the case of 5 users, and Doppler frequency is $50 \mathrm{~Hz}$

\section{ACKNOWLEDGMENTS}

This research was supported by the Vietnamese National Foundation for Science and Technology Development (NAFOSTED) under the project 10202-07-09.

\section{REFERENCES}

[1] Y. J. Zhang, Letaief: "An Efficient ResourceAllocation Scheme for Spatial Multiuser Access in MIMO/OFDM Systems" - IEEE Transactions on communications, Vol. 53, No. 1, January 2005107

[2] J. Jang and K. B. Lee, "Transmit Power Adaptation for Multiuser OFDM System,"'- IEEE Journal on Selected Areas in Communications, vol. 21, pp. 171-178, February 2003.

[3] Z. Shen, J. G. Andrews, and B. L. Evans, "Adaptive resource allocation in multiuser OFDM systems with proportional rate constraints" IEEE Transactions on Wireless Communications, vol. 4, no. 6, pp. $2726\{2737$, Nov. 2005.

[4] V. D. Nguyen, M. V. Pham and A. S. Dang: "Dynamic Sub-Channel Assignment for Cellular OFDMA Networks with Full Frequency-Reuse" - Post, Telecommunication and Information Technology Journal, Series 3, Issue 2, 12/2007

[5] C. Zeng, L. M. C. Hoo, J. M. Ciof.: "Effcient waterfilling algorithms for a Gaussian multiaccess channel with ISI', VTC Fall '00, Boston, USA, Sep. 2000.

[6] G. Münz, S. P.etschinger, J. Speidel, "An effcient waterfilling algorithm for multiple access OFDM", Globecom '02, Taipei, Taiwan, accepted for publication

[7] C. Y. Wong, R.S. Cheng, K.B. Letaief andR.D. Murch, "Multiuser OFDM with adaptive subcarrier, bit, and power allocation", IEEE JSAC, vol. 17, no. 10, Oct. 1999.

[8] ETSI DTS/BRAN-0023003 HIPERLAN Type 2 Technical Specification; Physical (PHY) layer. 1999.

[9] R. W. Heath, Jr.: "Antenna Selection for Spatial Multiplexing Systems with Linear Receivers" - IEEE Communications Letters, Vol. 5, No., April 2001 


\section{AUTHORS' BIOGRAPHIES}

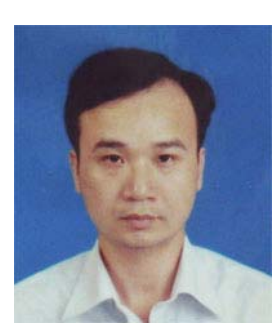

Quoc Khuong Nguyen received the Telecommunications Engineering degree in 1995 and Master degree in 1997 at the Hanoi University Of Technology (HUT) Vietnam. His research interests are in the field of wireless communications especially MIMO-OFDM systems, Signal Processing, DSP applications. He is currently a lecturer of HUT, and working towards his Ph.D. degree.

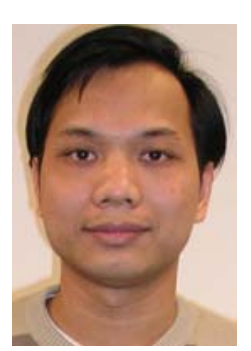

Van Duc Nguyen was born in Vietnam, in 1973. He received the Bachelor and Master of Engineering degrees in Electronics and Communications from the Hanoi University of Technology, Vietnam, in 1995 and 1997, respectively, and the Dr.-Eng. degree in Communications Engineering from the University of Hannover, Germany, in 2003.

His current research interests include Mobile Radio Communications, especially MIMO-OFDM systems, and radio resource management, channel coding for wireless networks.

Quoc Trung Nguyen is an associate professor of the Faculty of Electronic and Telecommunication, Hanoi University Of Technology. His research interests are in areas of Signal processing and wireless communication.
Dao Minh Hung was born in Binh

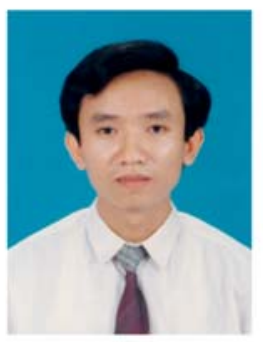
Dinh, Vietnam, on December 10, 1969. He received the Bachelor of Physics - Industrial Technique from the Quy Nhon college of teacher education in 1991, the Dipl. Eng. in Electronics and Communication, the M. Eng. in Electronics and Communication from the Technical University of Hanoi, Vietnam in 1998 and 2004 respectively. From 1991 to now, he has been working as a lecturer of the Quy Nhon University. His current research interests include Digital communications and MIMOOFDM system. 